\title{
A First-Order Predicate Calculus
}

\author{
Agata Darmochwal ${ }^{1}$ \\ Warsaw Uniwersity \\ Białystok
}

\begin{abstract}
Summary. A continuation of [3], with an axiom system of firstorder predicate theory. The consequence $\mathrm{Cn}$ of a set of formulas $X$ is defined as the intersection of all theories containing $X$ and some basic properties of it has been proved (monotonicity, idempotency, completness etc.). The notion of a proof of given formula is also introduced and it is shown that $\operatorname{Cn} X=\{p: p$ has a proof w.r.t. $X\}$. First 14 theorems are rather simply facts. I just wanted them to be included in the data base.
\end{abstract}

MML Identifier: CQC_THE1.

The papers [11], [10], [9], [8], [4], [6], [1], [5], [2], [7], and [3] provide the terminology and notation for this paper. In the sequel $i, j, n, k, l$ will be natural numbers. One can prove the following propositions:

(1) If $n \leq 0$, then $n=0$.

(2) If $n \leq 1$, then $n=0$ or $n=1$.

(3) If $n \leq 2$, then $n=0$ or $n=1$ or $n=2$.

(4) If $n \leq 3$, then $n=0$ or $n=1$ or $n=2$ or $n=3$.

(5) If $n \leq 4$, then $n=0$ or $n=1$ or $n=2$ or $n=3$ or $n=4$.

(6) If $n \leq 5$, then $n=0$ or $n=1$ or $n=2$ or $n=3$ or $n=4$ or $n=5$.

(7) If $n \leq 6$, then $n=0$ or $n=1$ or $n=2$ or $n=3$ or $n=4$ or $n=5$ or $n=6$.

(8) If $n \leq 7$, then $n=0$ or $n=1$ or $n=2$ or $n=3$ or $n=4$ or $n=5$ or $n=6$ or $n=7$.

(9) If $n \leq 8$, then $n=0$ or $n=1$ or $n=2$ or $n=3$ or $n=4$ or $n=5$ or $n=6$ or $n=7$ or $n=8$.

(10) If $n \leq 9$, then $n=0$ or $n=1$ or $n=2$ or $n=3$ or $n=4$ or $n=5$ or $n=6$ or $n=7$ or $n=8$ or $n=9$.

\footnotetext{
${ }^{1}$ Supported by RPBP.III-24.C1
} 
Next we state two propositions:

(11) $\{k: k \leq n+1\}=\{i: i \leq n\} \cup\{n+1\}$.

(12) For every $n$ holds $\{k: k \leq n\}$ is finite.

In the sequel $X, Y, Z$ denote sets. One can prove the following two propositions:

(13) If $X$ is finite and $X \subseteq[: Y, Z$ : , then there exist sets $A, B$ such that $A$ is finite and $A \subseteq Y$ and $B$ is finite and $B \subseteq Z$ and $X \subseteq[: A, B$ :].

(14) If $X$ is finite and $Z$ is finite and $X \subseteq[: Y, Z$ : , then there exists a set $A$ such that $A$ is finite and $A \subseteq Y$ and $X \subseteq[A, Z$ :

For simplicity we adopt the following convention: $T, S, X, Y$ will be subsets of $\mathrm{WFF}_{\mathrm{CQC}}, p, q, r, t, F$ will be elements of $\mathrm{WFF}_{\mathrm{CQC}}, s$ will be a formula, and $x, y$ will be bound variables. Let us consider $T$. We say that $T$ is a theory if and only if:

(i) VERUM $\in T$,

(ii) for all $p, q, r, s, x, y$ holds $(\neg p \Rightarrow p) \Rightarrow p \in T$ and $p \Rightarrow(\neg p \Rightarrow q) \in T$ and $(p \Rightarrow q) \Rightarrow(\neg(q \wedge r) \Rightarrow \neg(p \wedge r)) \in T$ and $p \wedge q \Rightarrow q \wedge p \in T$ but if $p \in T$ and $p \Rightarrow q \in T$, then $q \in T$ and $\forall_{x} p \Rightarrow p \in T$ but if $p \Rightarrow q \in T$ and $x \notin \operatorname{snb}(p)$, then $p \Rightarrow \forall_{x} q \in T$ but if $s(x) \in \mathrm{WFF}_{\mathrm{CQC}}$ and $s(y) \in \mathrm{WFF}_{\mathrm{CQC}}$ and $x \notin \operatorname{snb}(s)$ and $s(x) \in T$, then $s(y) \in T$.

Next we state a number of propositions:

(15) Suppose that

(i) $\quad$ VERUM $\in T$,

(ii) for all $p, q, r, s, x, y$ holds $(\neg p \Rightarrow p) \Rightarrow p \in T$ and $p \Rightarrow(\neg p \Rightarrow q) \in T$ and $(p \Rightarrow q) \Rightarrow(\neg(q \wedge r) \Rightarrow \neg(p \wedge r)) \in T$ and $p \wedge q \Rightarrow q \wedge p \in T$ but if $p \in T$ and $p \Rightarrow q \in T$, then $q \in T$ and $\forall_{x} p \Rightarrow p \in T$ but if $p \Rightarrow q \in T$ and $x \notin \operatorname{snb}(p)$, then $p \Rightarrow \forall_{x} q \in T$ but if $s(x) \in \mathrm{WFF}_{\mathrm{CQC}}$ and $s(y) \in \mathrm{WFF}_{\mathrm{CQC}}$ and $x \notin \operatorname{snb}(s)$ and $s(x) \in T$, then $s(y) \in T$.

Then $T$ is a theory.

(16) If $T$ is a theory, then VERUM $\in T$.

(17) If $T$ is a theory, then $(\neg p \Rightarrow p) \Rightarrow p \in T$.

(18) If $T$ is a theory, then $p \Rightarrow(\neg p \Rightarrow q) \in T$.

(19) If $T$ is a theory, then $(p \Rightarrow q) \Rightarrow(\neg(q \wedge r) \Rightarrow \neg(p \wedge r)) \in T$.

(20) If $T$ is a theory, then $p \wedge q \Rightarrow q \wedge p \in T$.

(21) If $T$ is a theory and $p \in T$ and $p \Rightarrow q \in T$, then $q \in T$.

(22) If $T$ is a theory, then $\forall_{x} p \Rightarrow p \in T$.

(23) If $T$ is a theory and $p \Rightarrow q \in T$ and $x \notin \operatorname{snb}(p)$, then $p \Rightarrow \forall_{x} q \in T$.

(24) If $T$ is a theory and $s(x) \in \mathrm{WFF}_{\mathrm{CQC}}$ and $s(y) \in \mathrm{WFF}_{\mathrm{CQC}}$ and $x \notin$ $\operatorname{snb}(s)$ and $s(x) \in T$, then $s(y) \in T$.

Let us consider $T, S$. Then $T \cup S$ is a subset of $\mathrm{WFF}_{\mathrm{CQC}}$. Then $T \cap S$ is a subset of $\mathrm{WFF}_{\mathrm{CQC}}$. Then $T \backslash S$ is a subset of $\mathrm{WFF}_{\mathrm{CQC}}$.

Let us consider $p$. Then $\{p\}$ is a subset of $\mathrm{WFF}_{\mathrm{CQC}}$.

Next we state the proposition 
(25) If $T$ is a theory and $S$ is a theory, then $T \cap S$ is a theory.

Let us consider $X$. The functor $\mathrm{Cn} X$ yielding a subset of $\mathrm{WFF}_{\mathrm{CQC}}$ is defined as follows:

$t \in \mathrm{Cn} X$ if and only if for every $T$ such that $T$ is a theory and $X \subseteq T$ holds $t \in T$.

We now state a number of propositions:

(26) $Y=\operatorname{Cn} X$ if and only if for every $t$ holds $t \in Y$ if and only if for every $T$ such that $T$ is a theory and $X \subseteq T$ holds $t \in T$.

(27) $\quad$ VERUM $\in \mathrm{Cn} X$.

(28) $\quad(\neg p \Rightarrow p) \Rightarrow p \in \operatorname{Cn} X$.

(29) $\quad p \Rightarrow(\neg p \Rightarrow q) \in \mathrm{Cn} X$.

(30) $\quad(p \Rightarrow q) \Rightarrow(\neg(q \wedge r) \Rightarrow \neg(p \wedge r)) \in \mathrm{Cn} X$.

(31) $\quad p \wedge q \Rightarrow q \wedge p \in \mathrm{Cn} X$.

(32) If $p \in \operatorname{Cn} X$ and $p \Rightarrow q \in \operatorname{Cn} X$, then $q \in \operatorname{Cn} X$.

(33) $\quad \forall_{x} p \Rightarrow p \in \operatorname{Cn} X$.

(34) If $p \Rightarrow q \in \operatorname{Cn} X$ and $x \notin \operatorname{snb}(p)$, then $p \Rightarrow \forall_{x} q \in \operatorname{Cn} X$.

(35) If $s(x) \in \mathrm{WFF}_{\mathrm{CQC}}$ and $s(y) \in \mathrm{WFF}_{\mathrm{CQC}}$ and $x \notin \operatorname{snb}(s)$ and $s(x) \in$ $\operatorname{Cn} X$, then $s(y) \in \operatorname{Cn} X$.

(36) $\operatorname{Cn} X$ is a theory.

(37) If $T$ is a theory and $X \subseteq T$, then $\operatorname{Cn} X \subseteq T$.

(38) $X \subseteq \operatorname{Cn} X$.

(39) If $X \subseteq Y$, then $\operatorname{Cn} X \subseteq \operatorname{Cn} Y$.

(40) $\operatorname{Cn}(\operatorname{Cn} X)=\operatorname{Cn} X$.

(41) $T$ is a theory if and only if $\operatorname{Cn} T=T$.

The non-empty set $\mathbb{K}$ is defined by:

$\mathbb{K}=\{k: k \leq 9\}$.

Next we state three propositions:

(42) $\mathbb{K}=\{k: k \leq 9\}$.

(43) $\quad 0 \in \mathbb{K}$ and $1 \in \mathbb{K}$ and $2 \in \mathbb{K}$ and $3 \in \mathbb{K}$ and $4 \in \mathbb{K}$ and $5 \in \mathbb{K}$ and $6 \in \mathbb{K}$ and $7 \in \mathbb{K}$ and $8 \in \mathbb{K}$ and $9 \in \mathbb{K}$.

(44) $\mathbb{K}$ is finite.

In the sequel $f, g$ are finite sequences of elements of $\left[\mathrm{WFF}_{\mathrm{CQC}}, \mathbb{K}:\right.$. The following proposition is true

(45) Suppose $1 \leq n$ and $n \leq \operatorname{len} f$. Then

(i) $(f(n))_{2}=0$, or

(ii) $(f(n))_{2}=1$, or

(iii) $\quad(f(n))_{\mathbf{2}}=2$, or

(iv) $\quad(f(n))_{\mathbf{2}}=3$, or

(v) $\quad(f(n))_{\mathbf{2}}=4$, or

(vi) $\quad(f(n))_{\mathbf{2}}=5$, or

(vii) $\quad(f(n))_{2}=6$, or 
(viii) $\quad(f(n))_{\mathbf{2}}=7$, or

(ix) $\quad(f(n))_{2}=8$, or

(x) $\quad(f(n))_{2}=9$.

Let $P R$ be a finite sequence of elements of : $\mathrm{WFF}_{\mathrm{CQC}}, \mathbb{K}:$, and let us consider $n, X$. Let us assume that $1 \leq n$ and $n \leq$ len $P R$. We say that $P R(n)$ is a correct proof step w.r.t. $X$ if and only if:

$(P R(n))_{\mathbf{1}} \in X$ if $(P R(n))_{\mathbf{2}}=0,(P R(n))_{\mathbf{1}}=\operatorname{VERUM}$ if $(P R(n))_{\mathbf{2}}=1$, there exists $p$ such that $(P R(n))_{\mathbf{1}}=(\neg p \Rightarrow p) \Rightarrow p$ if $(P R(n))_{\mathbf{2}}=2$, there exist $p, q$ such that $(P R(n))_{\mathbf{1}}=p \Rightarrow(\neg p \Rightarrow q)$ if $(P R(n))_{\mathbf{2}}=3$, there exist $p, q$, $r$ such that $(P R(n))_{\mathbf{1}}=(p \Rightarrow q) \Rightarrow(\neg(q \wedge r) \Rightarrow \neg(p \wedge r))$ if $(P R(n))_{\mathbf{2}}=4$, there exist $p, q$ such that $(P R(n))_{1}=p \wedge q \Rightarrow q \wedge p$ if $(P R(n))_{\mathbf{2}}=5$, there exist $p, x$ such that $(P R(n))_{\mathbf{1}}=\forall_{x} p \Rightarrow p$ if $(P R(n))_{\mathbf{2}}=6$, there exist $i, j$, $p, q$ such that $1 \leq i$ and $i<n$ and $1 \leq j$ and $j<i$ and $p=(P R(j))_{1}$ and $q=(P R(n))_{1}$ and $(P R(i))_{1}=p \Rightarrow q$ if $(P R(n))_{\mathbf{2}}=7$, there exist $i, p, q$, $x$ such that $1 \leq i$ and $i<n$ and $(P R(i))_{1}=p \Rightarrow q$ and $x \notin \operatorname{snb}(p)$ and $(P R(n))_{\mathbf{1}}=p \Rightarrow \forall_{x} q$ if $(P R(n))_{\mathbf{2}}=8$, there exist $i, x, y, s$ such that $1 \leq i$ and $i<n$ and $s(x) \in \mathrm{WFF}_{\mathrm{CQC}}$ and $s(y) \in \mathrm{WFF}_{\mathrm{CQC}}$ and $x \notin \operatorname{snb}(s)$ and $s(x)=(P R(i))_{\mathbf{1}}$ and $s(y)=(P R(n))_{\mathbf{1}}$ if $(P R(n))_{\mathbf{2}}=9$.

The following propositions are true:

(46) If $1 \leq n$ and $n \leq \operatorname{len} f$ and $(f(n))_{2}=0$, then $f(n)$ is a correct proof step w.r.t. $X$ if and only if $(f(n))_{1} \in X$.

(47) If $1 \leq n$ and $n \leq$ len $f$ and $(f(n))_{2}=1$, then $f(n)$ is a correct proof step w.r.t. $X$ if and only if $(f(n))_{1}=$ VERUM.

(48) If $1 \leq n$ and $n \leq$ len $f$ and $(f(n))_{2}=2$, then $f(n)$ is a correct proof step w.r.t. $X$ if and only if there exists $p$ such that $(f(n))_{1}=(\neg p \Rightarrow p) \Rightarrow p$.

(49) If $1 \leq n$ and $n \leq$ len $f$ and $(f(n))_{\mathbf{2}}=3$, then $f(n)$ is a correct proof step w.r.t. $X$ if and only if there exist $p, q$ such that $(f(n))_{1}=p \Rightarrow(\neg p \Rightarrow q)$.

(50) If $1 \leq n$ and $n \leq$ len $f$ and $(f(n))_{\mathbf{2}}=4$, then $f(n)$ is a correct proof step w.r.t. $X$ if and only if there exist $p, q, r$ such that $(f(n))_{1}=(p \Rightarrow$ $q) \Rightarrow(\neg(q \wedge r) \Rightarrow \neg(p \wedge r))$.

(51) If $1 \leq n$ and $n \leq$ len $f$ and $(f(n))_{2}=5$, then $f(n)$ is a correct proof step w.r.t. $X$ if and only if there exist $p, q$ such that $(f(n))_{1}=p \wedge q \Rightarrow q \wedge p$.

(52) If $1 \leq n$ and $n \leq$ len $f$ and $(f(n))_{2}=6$, then $f(n)$ is a correct proof step w.r.t. $X$ if and only if there exist $p, x$ such that $(f(n))_{1}=\forall_{x} p \Rightarrow p$.

(53) Suppose $1 \leq n$ and $n \leq \operatorname{len} f$ and $(f(n))_{\mathbf{2}}=7$. Then $f(n)$ is a correct proof step w.r.t. $X$ if and only if there exist $i, j, p, q$ such that $1 \leq i$ and $i<n$ and $1 \leq j$ and $j<i$ and $p=(f(j))_{1}$ and $q=(f(n))_{1}$ and $(f(i))_{1}=p \Rightarrow q$.

(54) Suppose $1 \leq n$ and $n \leq \operatorname{len} f$ and $(f(n))_{2}=8$. Then $f(n)$ is a correct proof step w.r.t. $X$ if and only if there exist $i, p, q, x$ such that $1 \leq i$ and $i<n$ and $(f(i))_{\mathbf{1}}=p \Rightarrow q$ and $x \notin \operatorname{snb}(p)$ and $(f(n))_{1}=p \Rightarrow \forall_{x} q$.

(55) Suppose $1 \leq n$ and $n \leq$ len $f$ and $(f(n))_{2}=9$. Then $f(n)$ is a correct proof step w.r.t. $X$ if and only if there exist $i, x, y, s$ such that $1 \leq i$ 
and $i<n$ and $s(x) \in \mathrm{WFF}_{\mathrm{CQC}}$ and $s(y) \in \mathrm{WFF}_{\mathrm{CQC}}$ and $x \notin \operatorname{snb}(s)$ and $s(x)=(f(i))_{1}$ and $(f(n))_{1}=s(y)$.

Let us consider $X, f$. We say that $f$ is a proof w.r.t. $X$ if and only if:

$f \neq \varepsilon$ and for every $n$ such that $1 \leq n$ and $n \leq \operatorname{len} f$ holds $f(n)$ is a correct proof step w.r.t. $X$.

The following propositions are true:

(56) $\quad f$ is a proof w.r.t. $X$ if and only if $f \neq \varepsilon$ and for every $n$ such that $1 \leq n$ and $n \leq$ len $f$ holds $f(n)$ is a correct proof step w.r.t. $X$.

(57) If $f$ is a proof w.r.t. $X$, then $\operatorname{rng} f \neq \emptyset$.

(58) If $f$ is a proof w.r.t. $X$, then $1 \leq \operatorname{len} f$.

(59) Suppose $f$ is a proof w.r.t. $X$. Then $(f(1))_{\mathbf{2}}=0$ or $(f(1))_{\mathbf{2}}=1$ or $(f(1))_{2}=2$ or $(f(1))_{2}=3$ or $(f(1))_{2}=4$ or $(f(1))_{2}=5$ or $(f(1))_{2}=6$.

(60) If $1 \leq n$ and $n \leq \operatorname{len} f$, then $f(n)$ is a correct proof step w.r.t. $X$ if and only if $f^{\frown} g(n)$ is a correct proof step w.r.t. $X$.

(61) If $1 \leq n$ and $n \leq \operatorname{len} g$ and $g(n)$ is a correct proof step w.r.t. $X$, then $f \frown g(n+\operatorname{len} f)$ is a correct proof step w.r.t. $X$.

(62) If $f$ is a proof w.r.t. $X$ and $g$ is a proof w.r.t. $X$, then $f^{\wedge} g$ is a proof w.r.t. $X$.

(63) If $f$ is a proof w.r.t. $X$ and $X \subseteq Y$, then $f$ is a proof w.r.t. $Y$.

(64) If $f$ is a proof w.r.t. $X$ and $1 \leq l$ and $l \leq \operatorname{len} f$, then $(f(l))_{1} \in \operatorname{Cn} X$.

Let us consider $f$. Let us assume that $f \neq \varepsilon$. The functor Effect $f$ yields an element of $\mathrm{WFF}_{\mathrm{CQC}}$ and is defined as follows:

Effect $f=(f(\operatorname{len} f))_{1}$.

The following propositions are true:

(65) If $f \neq \varepsilon$, then Effect $f=(f(\operatorname{len} f))_{\mathbf{1}}$.

(66) If $f$ is a proof w.r.t. $X$, then Effect $f \in \operatorname{Cn} X$.

(67) $X \subseteq\left\{F: \bigvee_{f}[f\right.$ is a proof w.r.t. $X \wedge$ Effect $\left.f=F]\right\}$.

(68) For every $X$ such that $Y=\left\{p: \bigvee_{f}[f\right.$ is a proof w.r.t. $X \wedge$ Effect $\left.f=p]\right\}$ holds $Y$ is a theory.

(69) For every $X$ holds $\left\{p: \bigvee_{f}[f\right.$ is a proof w.r.t. $\left.X \wedge \operatorname{Effect} f=p]\right\}=$ Cn $X$.

(70) $p \in \operatorname{Cn} X$ if and only if there exists $f$ such that $f$ is a proof w.r.t. $X$ and Effect $f=p$.

(71) If $p \in \operatorname{Cn} X$, then there exists $Y$ such that $Y \subseteq X$ and $Y$ is finite and $p \in \operatorname{Cn} Y$.

The subset $\emptyset_{\mathrm{CQC}}$ of $\mathrm{WFF}_{\mathrm{CQC}}$ is defined by:

$\emptyset_{\mathrm{CQC}}=\emptyset_{\mathrm{WFF}_{\mathrm{CQC}}}$.

We now state the proposition

(72) $\emptyset_{\mathrm{CQC}}=\emptyset_{\mathrm{WFF}_{\mathrm{CQC}}}$.

The subset Taut of $\mathrm{WFF}_{\mathrm{CQC}}$ is defined as follows:

Taut $=\mathrm{Cn} \emptyset_{\mathrm{CQC}}$. 
One can prove the following propositions:

(73) $\quad$ Taut $=\mathrm{Cn} \emptyset_{\mathrm{CQC}}$.

(74) If $T$ is a theory, then Taut $\subseteq T$.

(75) Taut $\subseteq$ Cn $X$.

(76) Taut is a theory.

(77) VERUM $\in$ Taut.

(78) $(\neg p \Rightarrow p) \Rightarrow p \in$ Taut.

(79) $\quad p \Rightarrow(\neg p \Rightarrow q) \in$ Taut.

(80) $\quad(p \Rightarrow q) \Rightarrow(\neg(q \wedge r) \Rightarrow \neg(p \wedge r)) \in$ Taut.

(81) $\quad p \wedge q \Rightarrow q \wedge p \in$ Taut.

(82) If $p \in$ Taut and $p \Rightarrow q \in$ Taut, then $q \in$ Taut.

(83) $\quad \forall_{x} p \Rightarrow p \in$ Taut.

(84) If $p \Rightarrow q \in$ Taut and $x \notin \operatorname{snb}(p)$, then $p \Rightarrow \forall_{x} q \in$ Taut.

(85) If $s(x) \in \mathrm{WFF}_{\mathrm{CQC}}$ and $s(y) \in \mathrm{WFF}_{\mathrm{CQC}}$ and $x \notin \operatorname{snb}(s)$ and $s(x) \in$ Taut, then $s(y) \in$ Taut.

Let us consider $X, s$. The predicate $X \vdash s$ is defined as follows:

$s \in \mathrm{Cn} X$.

Next we state a number of propositions:

(86) $X \vdash s$ if and only if $s \in \operatorname{Cn} X$.

(87) $X \vdash$ VERUM.

(88) $\quad X \vdash(\neg p \Rightarrow p) \Rightarrow p$.

(89) $\quad X \vdash p \Rightarrow(\neg p \Rightarrow q)$.

(90) $\quad X \vdash(p \Rightarrow q) \Rightarrow(\neg(q \wedge r) \Rightarrow \neg(p \wedge r))$.

(91) $\quad X \vdash p \wedge q \Rightarrow q \wedge p$.

(92) If $X \vdash p$ and $X \vdash p \Rightarrow q$, then $X \vdash q$.

(93) $X \vdash \forall_{x} p \Rightarrow p$.

(94) If $X \vdash p \Rightarrow q$ and $x \notin \operatorname{snb}(p)$, then $X \vdash p \Rightarrow \forall x q$.

(95) If $s(x) \in \mathrm{WFF}_{\mathrm{CQC}}$ and $s(y) \in \mathrm{WFF}_{\mathrm{CQC}}$ and $x \notin \operatorname{snb}(s)$ and $X \vdash s(x)$, then $X \vdash s(y)$.

Let us consider $s$. The predicate $\vdash s$ is defined as follows:

$\emptyset_{\mathrm{CQC}} \vdash s$.

Next we state two propositions:

(96) $\vdash s$ if and only if $\emptyset_{\mathrm{CQC}} \vdash s$.

(97) $\vdash s$ if and only if $s \in$ Taut.

Let us consider $s$. Let us note that one can characterize the predicate $\vdash s$ by the following (equivalent) condition: $s \in$ Taut.

We now state a number of propositions:

(98) If $\vdash p$, then $X \vdash p$.

(99) $\vdash$ VERUM. 
$(100)$

$$
\text { then } \vdash s(y) \text {. }
$$

\section{References}

[1] Grzegorz Bancerek. The fundamental properties of natural numbers. Formalized Mathematics, 1(1):41-46, 1990.

[2] Grzegorz Bancerek and Krzysztof Hryniewiecki. Segments of natural numbers and finite sequences. Formalized Mathematics, 1(1):107-114, 1990.

[3] Czesław Byliński. A classical first order language. Formalized Mathematics, 1(4):669-676, 1990.

[4] Czesław Byliński. Functions and their basic properties. Formalized Mathematics, 1(1):55-65, 1990.

[5] Agata Darmochwał. Finite sets. Formalized Mathematics, 1(1):165-167, 1990.

[6] Krzysztof Hryniewiecki. Basic properties of real numbers. Formalized Mathematics, 1(1):35-40, 1990.

[7] Piotr Rudnicki and Andrzej Trybulec. A first order language. Formalized Mathematics, 1(2):303-311, 1990.

[8] Andrzej Trybulec. Domains and their Cartesian products. Formalized Mathematics, 1(1):115-122, 1990.

[9] Andrzej Trybulec. Tuples, projections and Cartesian products. Formalized Mathematics, 1(1):97-105, 1990.

[10] Zinaida Trybulec. Properties of subsets. Formalized Mathematics, 1(1):67-71, 1990.

[11] Zinaida Trybulec and Halina Święczkowska. Boolean properties of sets. Formalized Mathematics, 1(1):17-23, 1990.

Received May 25, 1990 